\title{
Telepresence Technology for Production: From Manual to Automated Assembly
}

\author{
Marwan Radi ${ }^{1}$, Andrea Reiter ${ }^{1}$, Michael F. Zäh ${ }^{1}$, \\ Thomas Müller ${ }^{2}$, and Alois Knoll ${ }^{2}$ \\ 1 Institute for Machine Tools and Industrial Management (iwb) \\ \{marwan.radi, andrea.reiter, michael.zaeh\}@iwb.tum.de \\ http: / / www.iwb.tum.de \\ 2 Robotics and Embedded Systems, Institut für Informatik VI \\ \{muelleth, knoll\}@in.tum.de \\ http: / / www6.in.tum.de/ \\ Technische Universität München, D-85748 Garching, Germany
}

\begin{abstract}
In this paper a telepresence system for micro- and macroscopic assembly is presented. The proposed system integrates haptic devices on the operator side for reflecting gripping and collision forces as well as artificial scene information. Scaling of the position and force information is implemented to overcome the physical constraints of micro- as well as macroassembly tasks.

Furthermore, the paper introduces a flexible automation framework for teaching in action primitives, which the operator can then compose to incrementally complex assembly tasks.

This combination of a haptic telepresence approach with a user-friendly automation framework allows the manufacturer to cope with the challenges of todays industrial requirements.
\end{abstract}

Keywords: Telepresence Technology, Manual Assembly, Flexible Automated Assembly.

\section{Introduction}

Regarding the production process, assembly takes usually up to 70 percent of costs [1]. This is why efficient assembly strategies are an important issue in many industrial sectors. Efficiency is often achieved by automation, which is preferable compared to manual assembly regarding time, repeatability and accuracy.

Nevertheless, automation is just suitable for a very specific range of products, which can easily be handled and produced in high quantities. It may not be cost-efficient for production of small batches, which is meanwhile one of the important growing production trends to meet the demand of individualization and customization. Some of the challenges in production of such goods are flexibility and reconfigurability (e.g. the need of tool changeover and reprogramming the machines after each batch). In spite of significant advances in the field of artificial intelligence and industrial automation [2], human intelligence is far superior in terms of reasoning, language comprehension, vision, and ingenuity [3]. Therefore, people are still necessary to manage complex assembly activities for personalized and customized products. 
However, the ability of the human is hindered in some cases by physical barriers. For instance, the production of micro products demands fine motor skills and good visual perception to deal with highly precise components. Moreover, some materials can have adverse health effects on humans, e.g. radioactive materials or ones being too bulky to be ergonomically handled.

Considering these and other production trends in society and economy, it becomes evident that new assembly techniques are required. A promising solution to cope with the aforementioned challenges in manual assembly is the telepresence technology. Up to now, telepresence is rarely used in industrial applications, however recently researchers started to investigate the implementation of telepresence technology in these environments.

Okazaki et al. [4] designed a portable micro factory and an operator workplace equipped with a standard joystick without force feedback. For handling nano-objects a remotely controlled nano-manipulation system with haptic feedback [5]6] was developed. For other industrial sections, different robotic assist systems were designed with or without force feedback to manually guide industrial robots [7]8].

Considering small batch sizes, intuitively, performing an assembly task in a telepresent approach once while recording it, and replaying it several times makes sense. In contrast to a standard automation system, which requires time-consuming reprogramming, this flexible automation approach with telepresent teach-in takes just as long as the manually controlled telepresence task and exploits the superior human abilities.

In this paper, a novel promising approach to telepresent assembly applications ranging from individual prototypes to small lot sizes is proposed.

\section{Manual Telepresent Assembly Systems}

In this section, a generic telepresence system for the assembly of both micro- and macroscopic products is described (see Figure 1). The dominant characteristic is the reliance on readily available commercial components (e.g. personal computers and cameras) and standard industrial equipment (e.g. force/torque sensors (FTS) and manipulators).

Thus, the operator workplace consists of a haptic device and a monitor for visual feedback (see Figure 1). In order to make it easier for the human operator to understand the device's movement [9], it is preferred to have a simple input device, by which taskrelevant degrees of freedom are enabled. Therefore, a 2-DOF force feedback joystick is

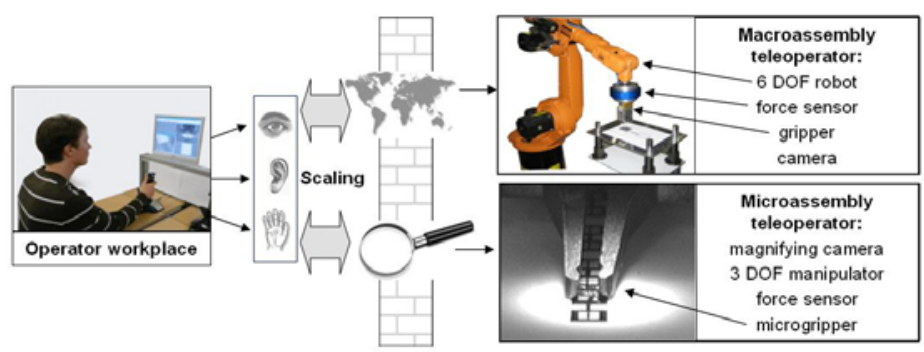

Fig. 1. Telepresence Systems for Micro and Macroscopic Assembly 
used in this setup as an Input/Output device. This device receives the motion commands from the human operator and sends them to the teleoperator side via a communication link. The signals are scaled bidirectionally, i.e., the displacement of the device to motions of the teleoperator and haptic feedback information back to the operator. To allow the human operator to steer the teleoperator in three degrees of freedom, a mapping strategy is implemented. The user can select which DOF to be moved by switching in between.

The teleoperator for the micro assembly, which is located in a clean room environment, consists of a high precision planar table ( $\mathrm{x}$ - and $\mathrm{y}$-axis) and a linear drive (z-axis) with an accuracy of $1.0 \mu \mathrm{m}$. A vacuum gripper and a magnifying camera are mounted onto the linear axis moving in the vertical direction. A one degree of freedom force sensor, positioned on the planar table, is used to measure forces in z-direction and thus detect contact between the microgripper and the table.

For the macroscopic assembly, an industrial robot is used as a teleoperator (see Figure 1). It is a 6-DOF articulated robot with a nominal payload of $6 \mathrm{~kg}$. The robot has a position controller with a real time communication interface, Ethernet Remote Sensor Interface (RSI). This RSI is used to connect the teleoperator with the central controller via the Ethernet TCP/IP protocol. For a pick and place task, a pneumatic gripper is mounted at the tool center point of the robot. A pan-tilt camera is used to give the human operator the visual feedback needed to accomplish the task.

\section{Towards Automation of Assembly Systems}

Although telepresence technology is considered a promising solution for manual assembly, it is not as profitable when switching from unique individual products to small batch sizes. Here, an approach combining the advantages of telepresence technology and automation techniques forms an efficient solution. Therefore, in this section a generic framework for flexible automation in the field of robot-assisted telepresent assembly is introduced.

\subsection{Framework Overview}

The framework presented here hence comprises a telepresence interface for convenient teach-in, and a graphical, user-friendly task automation facility. From a software engineering perspective, the framework is mainly built from three building blocks, namely the information storage, processing unit, and generic interface.

Generic Interface (GI). The first building block provides an easy-to-use interface abstraction for accessing external hardware components such as the real time connectors for robot control, grippers and servos, or image sensors, user IO-devices (mouse, keyboard, etc.), or other sensory devices, e.g. FTS.

However, implementing a generic interface is not limited to IO-devices, but indeed one can write an interface to virtually any external component, be it software or hardware. For instance considering software libraries, at the time of publication, the framework already provides predefined interfaces to the robust model-based realtime tracking library OpenTL [10] and the library underlying the efficient EET (exploring/ exploiting 
tree) planner [11] for advanced industrial robot control. Additionally, in order to support seamless integration with external applications, interfaces for accessing socket connectors for remote control, data exchange, and remote procedure calls are supplied by the framework, as well as an interface for running arbitrary executables.

Processing Unit (PU). The base class for processing data, the PU, provides a configuration, control and feedback facility as well as a possibility to share information with other PU's. Furthermore, each processing unit is designed as a thread and supplies a description of the action it performs to the automation system (see Section 3.2).

Typically, an application comprises a set of PUs, where PUs perform their action either continuously or they are triggered by an event. While most hardware interfaces need a cyclic, continuous update/ retrieval (such as the robot joint values or the camera interface), higher-level actions wrapped into a PU, e.g. moving the end-effector from $A$ to $B$, handing over a workpiece to the next robot, or finding a grasping point in a visual scene, most commonly need a trigger-event in the assembly workflow.

Information Storage (IS). In order to map a complex assembly workflow, exchanging data between PUs is essential. For example, the input device unit maps to the target pose generation unit, which again maps to the joint values corresponding to a robotic end-effector.

The singleton information storage supplied by the framework is the building block designed for this purpose. Figure 2 shows the workflow for data registration, storage, and retrieval modalities as a diagram.

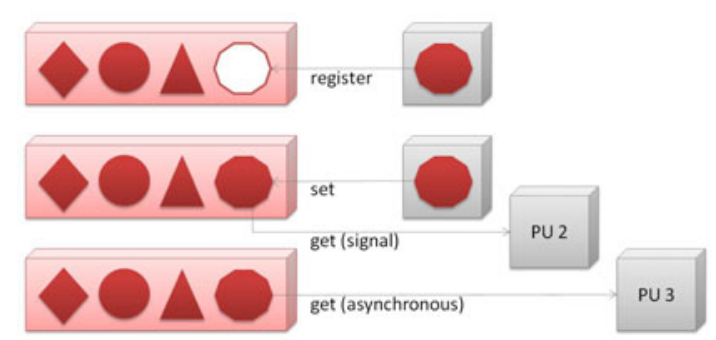

Fig. 2. Dataflow for a data item in the information storage

As shown in the figure, after registering a data-item, synchronous and asynchronous data access is possible, i.e. PUs can either listen for the event generated, when a data item has changed in the storage, or poll the data only on demand.

\subsection{Task Automation}

Tasks are generally described as a set of connected actions, where each processing unit defines a such action. In order to combine actions to a complex task, a generic action description comprising an identifier, IO- and configuration parameters, etc., was developed. Utilizing this action description, it is possible to specify a complex task by combination of primitive actions. These primitive actions, e.g., operations like "grasp", 
"screw", or "move-to $x y$ ", are teached in using the telepresence system described in Section 2 .

Furthermore, the framework supplies an automation unit, which enables a user to transform a task into an action and generate a new processing unit (see Figure 3) from it through graphical interaction.

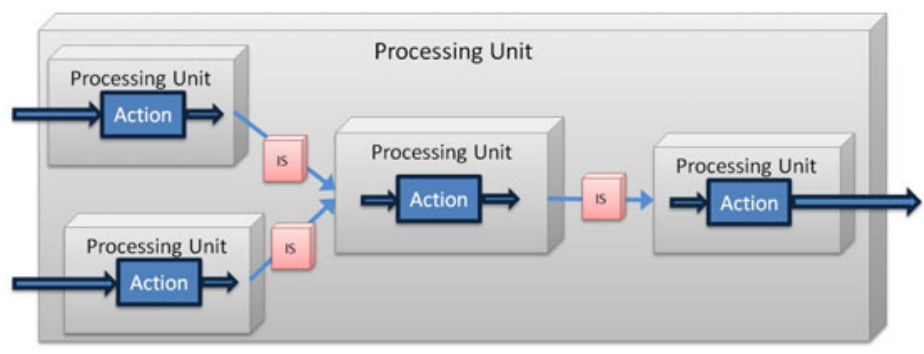

Fig. 3. Processing units can be generated online to combine a set of arbitrary actions into a more complex one for automation

These composite actions are then provided to the user interface for further combination, hence increasingly complex tasks can be automated. This enables the user to conveniently adapt a production system to a novel product variant requiring different assembly steps.

\section{Conclusion}

Addressing the challenges of manufacturing highly customized products, this paper presents a telepresence system tailored to micro- and macroscopic assembly tasks. The system integrates haptic feedback devices for reflecting gripping and collision forces as well as artificial scene information (virtual walls, etc.). Furthermore, the proposed system incorporates facilities to generate automation functions for small lot sizes, using the presented telepresence system for teach-in.

One can expect that the presented combination of the complementary techniques, namely telepresent assembly and flexible automation, will satisfy todays and future industrial requirements for highly adaptive production systems.

\section{Acknowledgements}

This work is supported by the German Research Foundation (DFG) within the Collaborative Research Center SFB 453 on "High-Fidelity Telepresence and Teleaction".

\section{References}

1. Chollet, S.K., Benmayor, L., Uehlinger, J.M., Jacot, J.: Cost effective micro-system assembly automation. In: Proceedings of 7th IEEE International Conference on Emerging Technologies and Factory Automation, ETFA 1999, vol. 1, pp. 359-366 (1999) 
2. Brooks, R.A., Aryananda, L., Edsinger, A., Fitzpatrick, P., Kemp, C., O’Reilly, U.M., TorresJara, E., Varshavskaya, P., Weber, J.: Sensing and manipulating built-for-human environments. International Journal of Humanoid Robotics 1(1), 1-28 (2004)

3. Nichol, Z., Liu, Y., Suchyta, P., Prokos, M., Goradia, A., Xi, N.: Super-media enhanced internet-based real-time teleoperation. In: Hands-On International Mechantronics and Automation Conference (2005)

4. Okazaki, Y., Mishima, N., Ashida, K.: Microfactory and micro machine tools. In: 1st KoreaJapan Conference on Positioning Technology, Daejeon, Korea, pp. 150-155 (2002)

5. Fahlbusch, S., Fatikow, S.: Micro force sensing in a micro robotic system. In: IEEE International Conference on Robotics and Automation, Seoul, Korea, pp. 3435-3440 (2001)

6. Kortschack, A., Shirinov, A., Trueper, T., Fatikow, S.: Development of mobile versatile nanohandling microrobots: design, driving principles, haptic control. Robotica 23(4), 419 434 (2005)

7. Schraft, R., Meyer, C., Parlitz, C., Helms, E.: Powermate - a safe and intuitive robot assistant for handling and assembly tasks. In: IEEE Proceedings of the Robotics and Automation, ICRA 2005 (2005)

8. Krüger, J., Bernhardt, R., Surdilovic, D.: Intelligent assist systems for flexible assembly. CIRP Annals 2006 55(1), 29-32 (2006)

9. Deml, B.: Human factors issues on the design of telepresence systems. Presence 16(5), 471487 (2007)

10. Panin, G., Lenz, C., Nair, S., Roth, E., Wojtczyk, M., Friedlhuber, T., Knoll, A.: A unifying software architecture for model-based visual tracking. In: Proc. of the 20th Annual Symposium of Electronic Imaging (2008)

11. Rickert, M., Brock, O., Knoll, A.: Balancing Exploration and Exploitation in Motion Planning. In: Proc. of the IEEE International Conference on Robotics and Automation (2008) 